\title{
LEISHMANIOSE TEGUMENTAR EM UM MUNICÍPIO DO NORTE DE MINAS GERAIS: ASPECTOS CLÍNICO-EPIDEMIOLÓGICOS E DISTRIBUIÇÃO ESPACIAL
}

\author{
Tegumentary Leishmaniasis in a municipality in the North of Minas Gerais: clinical- \\ epidemiological aspects and spatial distribution
}

\author{
Renata Luiz Ursine ${ }^{1}$ \\ Emanuelle de Moura Santos Xavier ${ }^{2}$ \\ Maria Suely Fernandes Gusmão ${ }^{3}$ \\ Hildeth Maisa Torres Farias ${ }^{4}$ \\ Marília Fonseca Rocha ${ }^{5}$ \\ Agna Soares da Silva Menezes ${ }^{6}$ \\ Renata Fiúza Damasceno ${ }^{7}$ \\ Ariela Ferreira Mota ${ }^{8}$ \\ Silvio Fernando Guimarães de Carvalho ${ }^{9}$ \\ Thallyta Maria Vieira ${ }^{10}$
}

\footnotetext{
${ }^{1}$ Universidade Estadual de Montes Claros (UNIMONTES), Programa de Pós-graduação em Ciências da Saúde. Montes Claros, MG- Brasil. Universidade Federal dos Vales do Jequitinhonha e Mucuri (UFVJM). Departamento de Ciências Biológicas. Diamantina MG- Brasil. $₫$ reursine@gmail.com@ https://orcid.org/0000-0003-0649-6708.

${ }^{2}$ Universidade Estadual de Montes Claros (UNIMONTES), Programa de Pós-graduação em Ciências da Saúde. Montes Claros, MG- Brasil. $\bowtie$ emanuellemsx88@ gmail.com. 1 https://orcid.org/0000-0003-0492-7391.

${ }^{3}$ Universidade Estadual de Montes Claros (UNIMONTES), Programa de Pós-graduação em Cuidados Primários em Saúde. Montes Claros, MG - Brasil. $\bowtie$ suelyfergusmao@ gmail.com. https://orcid.org/0000-0001-9736-8933.

${ }^{4}$ Universidade Federal dos Vales do Jequitinhonha e Mucuri (UFVJM), Programa de Pós-graduação em Saúde, Sociedade e Ambiente. Diamantina MG- Brasil. $\square$ maisinhafarias@gmail.com. 1 https://orcid.org/0000-0001-71181855 .

${ }^{5}$ Centro de Pesquisa René Rachou (FIOCRUZ), Doutorado em Ciências da Saúde. Belo Horizonte, MG - Brasil. Universidade Estadual de Montes Claros (UNIMONTES), Departamento de Saúde Mental e Saúde Coletiva. Montes Claros, MG- Brasil. $\square$ rfmarilia@ hotmail.com. 1 https://orcid.org/0000-0002-9793-2706.

${ }^{6}$ Universidade Estadual de Montes Claros (UNIMONTES), Programa de Pós-graduação em Ciências da Saúde. Montes Claros, MG- Brasil. Superintendência Regional de Saúde de Montes Claros. Montes Claros, MG- Brasil. $\square$ agna.menezes@saude.mg.gov.br. 1 https://orcid.org/0000-0003-2913-0071.

${ }^{7}$ Universidade Estadual de Montes Claros (UNIMONTES), Programa de Pós-graduação em Ciências da Saúde. Montes Claros, MG- Brasil. $\square$ damascenoenf@gmail.com. https://orcid.org/0000-0002-9525-8527.

${ }^{8}$ Universidade Estadual de Montes Claros (UNIMONTES), Programa de Pós-graduação em Ciências da Saúde. Montes Claros, MG- Brasil. $\square$ arielamota@hotmail.com. 1 https://orcid.org/0000-0002-2315-5318.

${ }^{9}$ Universidade Federal de Minas Gerais (UFMG), Doutorado em Ciências da Saúde. Belo Horizonte, MG- Brasil. Universidade Estadual de Montes Claros (UNIMONTES), Programa de Pós-graduação em Ciências da Saúde. Montes Claros, MG- Brasil. $₫$ guimaraescarvalho@yahoo.com.br. 1 https://orcid.org/0000-0002-6819-8418.

${ }^{10}$ Universidade Federal de Minas Gerais (UFMG), Doutorado em Parasitologia. Belo Horizonte, MG- Brasil. Universidade Estadual de Montes Claros (UNIMONTES), Programa de Pós-Graduação em Ciências da Saúde. Montes Claros, MG- Brasil. $\bowtie$ thallyta.vieira@ unimontes.br. 1 https://orcid.org/0000-0002-1483-6234.
}

$\begin{array}{ccc}\text { Recebido em } & \text { Aceito em } & \text { Publicado em } \\ 24 / 06 / 2021 & 12 / 08 / 2021 & 21 / 10 / 2021\end{array}$


Resumo: Objetivos: Analisar aspectos clínicos, epidemiológicos e a distribuição espacial da Leishmaniose Tegumentar em um município endêmico do Brasil (2012-2019). Métodos: Estudo epidemiológico, transversal e descritivo, realizado a partir de dados de Leishmaniose Tegumentar registrados no Sistema de Informação de Agravos de Notificação e no Sistema de Informação de Mortalidade. A análise estatística descritiva contemplou variáveis sociodemográficas e variáveis referentes à forma clínica, tratamento e evolução do caso. A análise de distribuição espacial foi realizada no software QGIS 3.4. Resultados: Foram registrados 380 casos de Leishmaniose Tegumentar, sendo a maioria dos pacientes do sexo masculino, adultos, que residiam na zona urbana. A principal forma de manifestação clínica foi cutânea $(93,9 \%)$; o antimonial pentavalente foi a droga de primeira escolha administrada em 75,5\% dos pacientes; $95,0 \%$ dos casos evoluíram para cura; $1,3 \%$ evoluíram para óbito (idade média: 61,8 anos; $60 \%$ possuíam coinfecção com HIV). As maiores taxas de incidência foram encontradas na região Oeste da cidade. Conclusões: No município estudado, diferentemente da maioria das regiões do Brasil, a Leishmaniose Tegumentar apresentou um perfil de ocorrência predominantemente urbano. Espera-se que este estudo possa contribuir com um melhor entendimento da epidemiologia desta doença e que possa nortear a elaboração de estratégias de controle.

Palavras-chave: Perfil Epidemiológico; Análise Espacial; Leishmaniose Cutânea; Sistema de Informação em Saúde.

Abstract: Objectives: To analyze clinical, epidemiological and spatial distribution aspects of Tegumentary Leishmaniasis in an endemic city in Brazil (2012-2019). Methods: Epidemiological, cross-sectional and descriptive study, based on data on Tegumentary Leishmaniasis registered in the Notifiable Diseases Information System and in the Mortality Information System. Descriptive statistical analysis included sociodemographic variables and variables related to the clinical form, treatment and evolution of the case. The spatial distribution analysis was performed using QGIS 3.4 software. Results: A total of 380 cases of Tegumentary Leishmaniasis were registered, most of the patients were male, adults, living in the urban area. The main form of clinical manifestation was cutaneous (93.9\%); pentavalent antimony was the drug of first choice administered to $75.5 \%$ of patients; $95.0 \%$ of cases evolved to cure; $1.3 \%$ progressed to death (mean age: 61.8 years; $60 \%$ had co-infection with HIV). The highest incidence rates were found in the western region of the city. Conclusions: In the municipality studied, unlike most regions in Brazil, Tegumentary Leishmaniasis presented a predominantly urban occurrence profile. It is hoped that this study can contribute to a better understanding of the epidemiology of this disease and that it can guide the development of control strategies.

Keywords: Health Profile; Spatial Analysis; Leishmaniasis, Cutaneous; Health Information Systems. 


\section{INTRODUÇÃO}

A Leishmaniose Tegumentar (LT) é uma doença infecto parasitária causada por uma variedade de protozoários do gênero Leishmania que acometem a pele e mucosas ${ }^{1}$. Segundo a Organização Mundial de Saúde (OMS), anualmente, registram-se aproximadamente 1,5 milhão de casos novos da LT no mundo ${ }^{2}$. Trata-se de uma doença tropical negligenciada de elevado impacto psicossocial, já que em torno de 40 milhões de pessoas no mundo sofrem com os efeitos estigmatizantes provocados pelas cicatrizes inativas da doença ${ }^{3}$.

Entre os dez países com o maior número de casos da LT no mundo, quatro estão nas Américas: Brasil, Colômbia, Nicarágua e Peru². No Brasil, há registros da doença em todas as unidades federativas, porém, com diferentes padrões de transmissão ${ }^{4,5}$. Na região Sudeste, o Estado de Minas Gerais corresponde a uma das principais áreas de ocorrência da LT. No período de 2007 a 2015, Minas Gerais apresentou taxa média de incidência de 6,1 casos/100.000 habitantes ${ }^{6}$, sendo a região Norte uma das áreas de maior ocorrência da LT no estado $^{6,7,8}$.

Pesquisas sobre a LT que integrem dados clínicos e epidemiológicos com ferramentas de análise espacial podem contribuir com o avanço do conhecimento científico sobre a doença de forma contextualizada, e servir como subsídio para implementação de estratégias de prevenção, vigilância e controle ${ }^{8}$. Neste contexto, este estudo objetivou analisar os aspectos clínicos, epidemiológicos e a distribuição espacial da LT no município de Montes Claros, Norte de Minas Gerais, no período de 2012 a 2019.

\section{MÉTODO}

Trata-se de um estudo epidemiológico, transversal e descritivo, realizado no município de Montes Claros, Norte de Minas Gerais, Brasil. Montes Claros é o quinto maior município do Estado de Minas Gerais em extensão territorial, ocupando uma área de $3.568,941 \mathrm{~km}^{2}$, apresenta população estimada de 413.487 mil habitantes e alto Índice de Desenvolvimento Humano Municipal $(0,770)^{9}$.

Os dados de notificações de LT analisados neste estudo foram coletados nos bancos de dados do Sistema de Informação de Agravos de Notificação (SINAN) e do Sistema de Informação de Mortalidade (SIM) da Secretaria Municipal de Saúde de Montes Claros 
(SMS/Montes Claros) após autorização da Secretaria Municipal de Saúde. A população do estudo foi constituída pelo total de casos de LT notificados no município e confirmados por critérios clínico-epidemiológicos e laboratorial no período de 2012 a 2019.

No SINAN foram coletados os seguintes dados: data de notificação, características sociodemográficas (sexo, zona de residência, idade, cor, escolaridade, endereço e ocupação), classificação epidemiológica do caso, forma de entrada, forma de manifestação clínica, métodos de diagnóstico, critério de confirmação, medicamentos administrados e evolução do caso. Foram excluídos deste estudo os casos cujas fichas de notificação apresentavam inconsistências e os casos que tiveram alteração no diagnóstico. No banco de dados do SIM, foram coletados dados referentes à causa básica e às causas contribuintes dos óbitos por LT.

A análise estatística foi realizada por meio do programa Statistical Package for the Social Sciences (SPSS), versão 22.0. Nessa etapa, foram calculadas frequências absolutas, frequências relativas e medidas de tendência central. O software QGIS 3.4 foi utilizado para realizar o georreferenciamento dos casos de LT e para construção dos mapas. A taxa de incidência da LT foi calculada por região de planejamento da cidade (Prefeitura de Montes Claros, 2016) em dois quadriênios (2012 a 2015; 2016 a 2019), considerando o seguinte cálculo:

$$
\mathrm{TX}=\frac{\mathrm{C}}{\mathrm{H}} * 10.000
$$

De forma que:

$\mathrm{TX}=$ taxa de incidência;

$\mathrm{C}=$ número de casos de LT no período analisado;

$\mathrm{H}=$ soma da população residente nos setores censitários enquadrados em cada região de planejamento, segundo o censo de $2010^{10}$.

Adotou-se a análise em quadriênios a fim de reduzir a variabilidade no número de casos da doença notificados entre os anos do estudo.

Aspectos éticos - Este estudo foi aprovado pelo Comitê de Ética em Pesquisa da Universidade Federal dos Vales do Jequitinhonha e Mucuri (UFVJM), sob o parecer 3.121.749, respeitando a Resolução 466/12 do Conselho Nacional da Saúde ${ }^{11}$. 


\section{RESULTADOS}

No período de 2012 a 2019, foram registrados 380 casos confirmados de LT no município de Montes Claros, com a predominância de ocorrência da doença em pessoas do sexo masculino $(62,4 \%)$, residentes da zona urbana $(78,4 \%)$. A maioria dos indivíduos acometidos pela LT era adulto $(57,1 \%)$, com idade media de 57,4 anos e se autodeclarava pardo $(59,7 \%)$ (Tabela 1$)$.

Tabela 1- Perfil sociodemográfico dos pacientes com Leishmaniose Tegumentar em Montes Claros, Minas Gerais, Brasil, entre 2012-2019.

\begin{tabular}{|c|c|c|c|}
\hline \multirow[t]{2}{*}{ Variáveis sociodemográficas } & \multicolumn{2}{|c|}{ Sexo } & \multirow{2}{*}{$\begin{array}{l}\text { Total } \\
\mathrm{n}=380\end{array}$} \\
\hline & $\begin{array}{c}\text { Feminino } \\
(\mathrm{n}=143)\end{array}$ & $\begin{array}{c}\text { Masculino } \\
(\mathrm{n}=237)\end{array}$ & \\
\hline \multicolumn{4}{|l|}{ Zona de residência } \\
\hline Rural & 31 & 51 & $82(21,6 \%)$ \\
\hline Urbana & 112 & 186 & $298(78,4 \%)$ \\
\hline \multicolumn{4}{|l|}{ Faixa etária } \\
\hline Criança (0 a 14) & 18 & 22 & $40(10,5 \%)$ \\
\hline Adolescente (15 a 19) & 05 & 18 & $23(06,1 \%)$ \\
\hline Adulto (20 a 59) & 80 & 137 & $217(57,1 \%)$ \\
\hline Idoso (60 ou mais) & 40 & 60 & $100(26,3 \%)$ \\
\hline \multicolumn{4}{|l|}{ Cor ou raça autodeclarada } \\
\hline Amarela $*$ & 00 & 04 & $04(01,0 \%)$ \\
\hline Branca & 42 & 59 & $101(26,6 \%)$ \\
\hline Parda & 87 & 140 & $227(59,7 \%)$ \\
\hline Preta & 05 & 17 & $22(05,8 \%)$ \\
\hline Ignorado & 08 & 17 & $25(06,6 \%)$ \\
\hline Indígena & 01 & 00 & $01(00,3 \%)$ \\
\hline \multicolumn{4}{|l|}{ Escolaridade } \\
\hline Analfabeto & 01 & 01 & $02(00,5 \%)$ \\
\hline Ensino Fundamental & 35 & 59 & $94(24,7 \%)$ \\
\hline Ensino Médio & 35 & 58 & $93(24,5 \%)$ \\
\hline Ensino Superior & 09 & 11 & $20(05,3 \%)$ \\
\hline Ignorado & 54 & 97 & $151(39,7 \%)$ \\
\hline Não se aplica & 9 & 11 & $20(05,3 \%)$ \\
\hline \multicolumn{4}{|l|}{ Ocupação } \\
\hline Atividades domésticas $* *$ & 59 & 32 & $91(24,0 \%)$ \\
\hline Atividades administrativas/comerciais & 09 & 26 & $35(09,2 \%)$ \\
\hline Estudante & 16 & 30 & $46(12,1 \%)$ \\
\hline Trabalhador agropecuário & 07 & 34 & $41(10,8 \%)$ \\
\hline Trabalhador da construção civil & 00 & 12 & $12(03,2 \%)$ \\
\hline Outros & 09 & 25 & $34(08,9 \%)$ \\
\hline Não se aplica ( $\leq 5$ anos) & 03 & 08 & $11(02,9 \%)$ \\
\hline Ignorado & 40 & 70 & $110(28,9 \%)$ \\
\hline
\end{tabular}


Fonte: Sistema de Informação de Agravos de Notificação (SINAN) da Secretaria Municipal de Saúde de Montes Claros. * Amarela: pessoa que se declarou de origem oriental: japonesa, chinesa ou coreana. **Atividades domésticas: dona de casa, empregada doméstica, faxineira, bem como aposentados (as), pensionistas e desempregados.

No que diz respeito à classificação epidemiológica, 345 (90,8\%) casos eram autóctones, 22 (5,8\%) indeterminados e $13(3,4 \%)$ importados. Quanto à forma de entrada no sistema, $356(93,7 \%)$ eram casos novos, $18(4,7 \%)$ recidivas e uma transferência $(0,3 \%)$. Em cinco $(1,3 \%)$ fichas de notificação essa informação foi ignorada.

A principal forma de manifestação clínica da LT foi a cutânea 357 (93,9\%), mas $17(4,5 \%)$ pacientes apresentaram a forma mucosa, quatro $(1,1 \%)$ a forma cutâneo-mucosa e em duas fichas $(0,5 \%)$ essa informação foi ignorada. A histopatologia foi o exame mais utilizado como subsídio para o diagnóstico dos pacientes $(57,6 \%)$, seguido do exame parasitológico direto (50,8\%). Informações complementares relacionadas ao diagnóstico e ao tratamento estão descritas na (Tabela 2).

Tabela 2- Aspectos relacionados ao diagnóstico e ao tratamento de pacientes que tiveram LT, residentes no município de Montes Claros, Minas Gerais (2012-2019).

\begin{tabular}{lc}
\hline Variáveis & $\mathrm{N}(\%)$ \\
\hline Exame Parasitológico & $160(42,1 \%)$ \\
Positivo & $33(08,7 \%)$ \\
Negativo & $187(49,2 \%)$ \\
Não realizado & \\
Intra Dermorreação de Montenegro (IDRM) & $130(34,2 \%)$ \\
Positivo & $24(06,3 \%)$ \\
Negativo & $226(59,5 \%)$ \\
Não realizado & \\
Histopatologia & $92(24,2 \%)$ \\
Encontro do parasita & $105(27,6 \%)$ \\
Compatível & $22(05,8 \%)$ \\
Não compatível & $161(42,4 \%)$ \\
Não realizado & \\
Critério de confirmação & $357(93,9 \%)$ \\
Laboratorial & $23(06,1 \%)$ \\
Clínico epidemiológico &
\end{tabular}


Antimonial Pentavalente

$287(75,5 \%)$

Anfotericina B

$77(20,3 \%)$

Outras (não especificada)

$8(02,1 \%)$

Ignorado

$8(02,1 \%)$

Outra Droga utilizada, na falência do tratamento inicial*

Anfotericina B

$79(84,0 \%)$

Isotionato de pentamidina

$8(08,5 \%)$

Outra (não especificada)

$7(07,5 \%)$

*Um total de 94 pacientes não apresentaram resposta satisfatória a primeira droga administrada, todos estes tendo realizado o tratamento inicial com o Antimonial Pentavalente.

Em relação à evolução dos casos, de acordo com as informações do SINAN, 361 $(95,0 \%)$ evoluíram para cura, dois $(0,5 \%)$ abandonaram o tratamento e cinco $(1,3 \%)$ evoluíram para óbito, sendo que destes dois foram classificados como óbito por LT e três como óbitos por outras causas. Destaca-se ainda que em 12 (3,2\%) fichas de notificação essa informação foi ignorada. A idade média dos pacientes que evoluíram para óbito foi de 61,8 anos $(\sigma=8,23)$, sendo que todos eram do sexo masculino. Informações complementares sobre a causa da morte, causas contribuintes e comorbidades associadas aos pacientes que morreram, de acordo com as informações do SINAN e do SIM estão apresentadas no (Quadro $1)$.

Quadro 1- Causa da morte e comorbidades associadas dos pacientes notificados com Leishmaniose Tegumentar no município de Montes Claros, Minas Gerais, Brasil (2012-2019) de acordo com as informações do SINAN e do SIM.

\begin{tabular}{|c|c|c|}
\hline $\begin{array}{l}\text { Forma de } \\
\text { manifestação clínica }\end{array}$ & SINAN & SIM \\
\hline Cutânea & $\begin{array}{l}\text { Óbito por } L T \\
\text { Co-infecção com HIV }\end{array}$ & $\begin{array}{l}\text { Causa Básica: Leishmaniose cutânea. } \\
\text { Causas contribuintes: Hipertensão } \\
\text { arterial e Doença cardíaca hipertensiva. }\end{array}$ \\
\hline Cutânea & Óbito por $L T$ & $\begin{array}{l}\text { Causa Básica: Peritonite aguda. Causas } \\
\text { contribuintes: LT, insuficiência renal. }\end{array}$ \\
\hline Cutânea & $\begin{array}{l}\text { Óbito por outras causas } \\
\text { Causas contribuintes: } \\
\text { Administração de outros } \\
\text { antibióticos administrado } \\
\text { por via sistêmica. }\end{array}$ & $\begin{array}{l}\text { Causa Básica: Insuficiência cardíaca. } \\
\text { Causas contribuintes: Administração de } \\
\text { outros antibióticos administrado por via } \\
\text { sistêmica. }\end{array}$ \\
\hline
\end{tabular}


Cutâneo-mucosa

Óbito por outras causas Co-infecção com HIV
Causa Básica: Doença pelo HIV resultando em encefalopatia. Causas contribuintes: LT e insuficiência renal aguda.

Causa Básica: Doença pelo HIV resultando em sarcoma de kaposi.

Fonte: Secretaria Municipal de Saúde.

Quanto à distribuição espacial da doença, verificou-se que em todas as regiões de planejamento da cidade houve o registro de casos da LT. No entanto, as maiores taxas de incidência, nos períodos analisados neste estudo, foram observadas nas áreas periféricas da região Oeste da cidade (Figura 1).

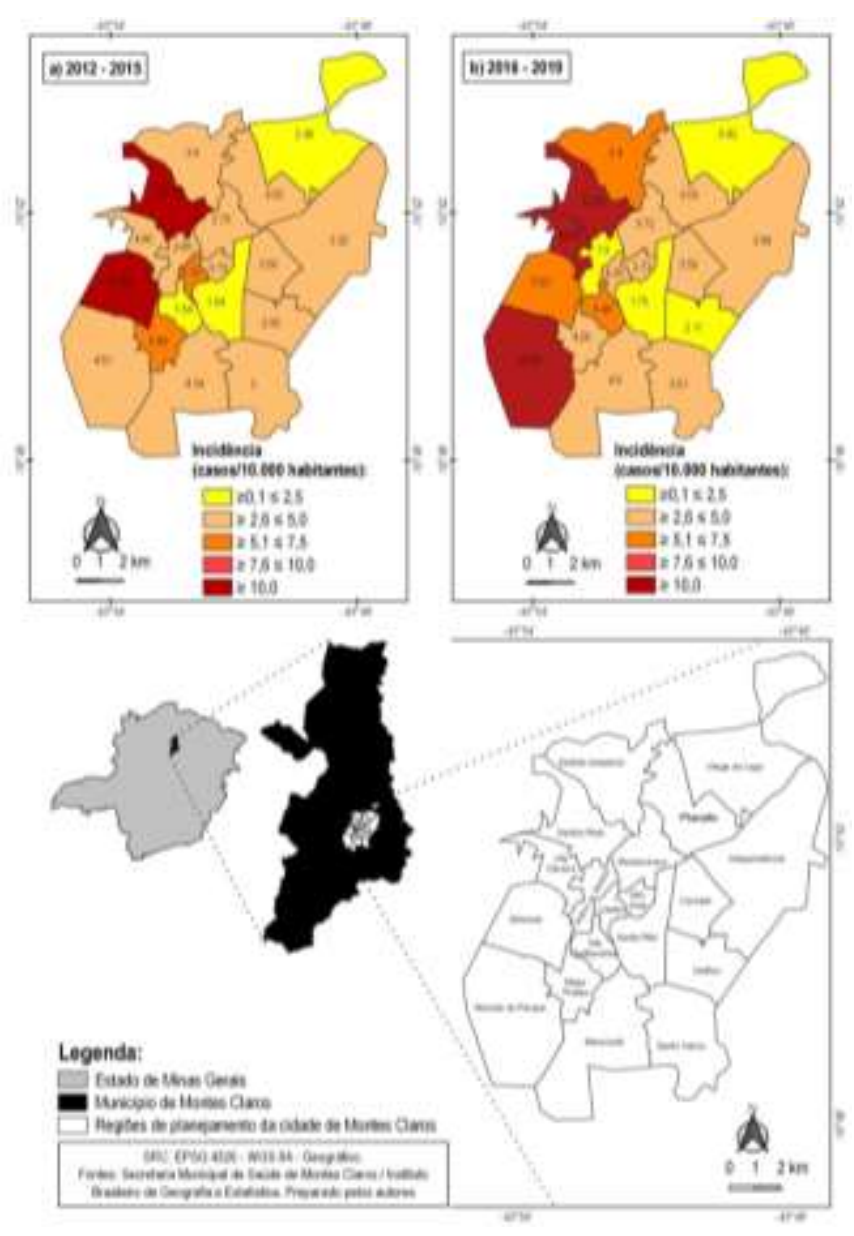

Figura 1 - Incidência da Leishmaniose Tegumentar, por regiões de planejamento da cidade de Montes Claros, Minas Gerais, no período de 2012 a 2019. 


\section{DISCUSSÃO}

No município de Montes Claros, a LT apresentou perfil de ocorrência predominantemente urbano, com maior acometimento de indivíduos do sexo masculino, adultos e autodeclarados pardos. Estudos realizados anteriormente nesse mesmo município também identificaram perfil de ocorrência urbano da $\mathrm{LT}^{12,13}$, o que difere do observado na maioria das outras regiões do Brasil ${ }^{14}$.

O maior acometimento de indivíduos adultos e do sexo masculino pode ser decorrente de exposição ocupacional, como atividades ligadas à agricultura, as quais são exercidas principalmente por indivíduos do sexo masculino ${ }^{15,16}$. Além disso, existem fortes evidências da maior predisposição dos homens a desenvolverem a doença em decorrência de fatores biológicos (hormonais) ${ }^{17}$. O predomínio de pessoas autodeclaradas como pardas na população estudada pode ser reflexo da própria composição racial do município. De acordo com dados do IBGE, cerca de 58,7\% da população de Montes Claros é composta por indivíduos pardos 9 .

Embora a maioria dos casos de LT neste estudo tenha sido classificada quanto à forma de entrada como caso novo, destaca-se o registro de 18 casos de recidivas. $\mathrm{O}$ mecanismo de recidiva da LT ainda é pouco compreendido ${ }^{18}$, mas pesquisadores já mostraram que o parasita persiste em cicatrizes das lesões mesmo após a cura clínica ${ }^{19}$. No entanto, também deve ser considerada a possibilidade de reinfecção, uma vez que o município de Montes Claros é endêmico para $\mathrm{LT}^{5,13}$.

No que se refere à forma de manifestação clínica, verificou-se o predomínio da forma cutânea, assim como observado em outros estudos ${ }^{15,20}$. O principal critério utilizado para o diagnóstico foi o laboratorial, sendo a histopatologia o exame mais utilizado. A baixa detecção de parasitas nos exames histológicos registrados pode ser decorrente do tempo de evolução da doença. Segundo Saldanha et. $a l^{21}$ há mais parasitas em biópsias de pacientes com lesões recentes do que com lesões tardias. Além disso, sabe-se que a sensibilidade dos exames pode variar de acordo com a capacidade técnica, qualidade do equipamento, dos insumos utilizados e as formas clínicas ${ }^{5}$. 
A morbidade associada a LT é alta, no entanto, a taxa de mortalidade é baixa, assumida como nula em alguns estudos ${ }^{3,4}$. Desta forma, a evolução da maioria dos pacientes tratados para a cura era esperada. Entre os pacientes que faleceram, 60\% apresentavam coinfecção com o HIV, condição crescentemente observada e que consiste em uma das causas de redução de sobrevida dos pacientes com LT $^{23,24}$. Além disso, a maioria dos pacientes que faleceu apresentava insuficiência renal e/ou insuficiência cardíaca. Sabe-se que as drogas utilizadas no tratamento da LT manifestam alta nefrotoxicidade e cardiotoxicidade ${ }^{5}$, condições que podem ter contribuído para a morte desses pacientes.

Ao realizar o pareamento das informações relacionadas aos óbitos por LT em Montes Claros no SINAN e no SIM, foram identificadas divergências e ausência de informações de relevância clínica nos dois sistemas de informação, o que demonstra a necessidade de implementar, no município, medidas para gestão da qualidade dos dados dos sistemas de informação em saúde. Ressalta-se que a integração das informações contidas no SINAN e no SIM foi fundamental para compreender os fatores que possivelmente contribuíram para a evolução do óbito nos pacientes com LT.

Neste estudo, as maiores taxas de incidência de LT foram observadas nas áreas periféricas da região Oeste do município. Estudo realizado em anos anteriores também identificou altas taxas de incidência da doença nessas áreas e uma autocorrelação positiva significativa entre a incidência da LT com o Índice de Vegetação da Diferença Normalizada (Normalized Difference Vegetation Index - NDVI) ${ }^{13}$. Desta forma, possivelmente os remanescentes de mata nativa e a proximidade com importantes reservas florestais, como o Parque Estadual da Lapa Grande e a Serra da Sapucaia esteja contribuindo com a maior incidência da doença nestas áreas.

Este estudo apresenta como limitação o vies de informação, referente ao uso de dados secundários, e neste contexto a possibilidade de subnotificação deve ser considerada. Além disso, os resultados do presente estudo devem ser interpretados considerando que se trata de um estudo transversal, que não possibilita estabelecer causalidade. Contudo, apesar dessas limitações, ele foi realizado em um município emblemático para regiões endêmicas para LT no Estado de Minas Gerais e utilizou ferramentas da análise espacial para integração de dados epidemiológicos. 


\section{CONSIDERAÇÕES FINAIS}

A LT apresentou um perfil de ocorrência predominantemente urbano no município de Montes Claros. A análise da distribuição espacial mostrou alta taxa de incidência de casos na região Oeste da cidade nos dois intervalos de período investigado, o que sugere a manutenção de condições favoráveis à ocorrência da LT nesta região. Neste contexto, faz-se necessário a realização ações integradas de infra-estrutura urbana e manejo ambiental a fim alterar as condições que propiciem o estabelecimento de vetores e reservatórios, e ações de educação em saúde nos territórios para promover a vigilância sustentada da doença.

Espera-se que este estudo possa contribuir com um melhor entendimento da epidemiologia da LT em uma importante area endêmica. E que também possa servir como subsídio para a elaboração de políticas de saúde para o controle desta doença.

\section{REFERÊNCIAS}

1- AKHOUNDI, Mohammad et al. A Historical Overview of the Classification, Evolution, and Dispersion of Leishmania Parasites and Sandflies. PLOS Neglected Tropical Diseases, San Francisco, v.10, n.3, p.1-40, 2016. Disponível em: https://doi.org/10.1371/journal.pntd.0004349. Acesso em: 20 abril de 2021.

2- WORLD HEALTH ORGANIZATION (WHO). Leishmaniasis [internet]. Geneva, Switzerland: $\quad$ WHO; $2019 . \quad$ Disponível https://www.paho.org/en/topics/leishmaniasis/cutaneous-and-mucosal-leishmaniasis. Acesso em: 05 de dezembro de 2020.

3- BAILEY, Freddie et al. A new perspective on cutaneous leishmaniasis-Implications for global prevalence and burden of disease estimates. PLOS Neglected Tropical Diseases, San Francisco, v.11, n.8, p.1-5, 2017. Disponível em: https://doi.org/10.1371/journal.pntd.0005739. Acesso em: 20 abril de 2021.

4- BEZERRA, Juliana Maria Trindade et al. Burden of leishmaniasis in Brazil and federated units, 1990-2016: Findings from Global Burden of Disease Study 2016. PLOS Neglected Tropical Diseases, San Francisco, v. 12, n.9, p.1-19, 2018. Disponível em: https://doi.org/10.1371/journal.pntd.0006697. Acesso em: 16 de abril de 2021.

5- BRASIL. Ministério da Saúde. Secretaria de Vigilancia em Saúde. Departamento de Vigilancia das Doenças Transmissíveis. Manual de vigilância da leishmaniose tegumentar. Brasília, Distrito Federal: Ministério da Saúde, 2017.189 p.

6- CARDOSO, Diogo Tavares et al. Identification of priority areas for surveillance of cutaneous leishmaniasis using spatial analysis approaches in Southeastern Brazil. BMC Infectious Diseases, London, v.19, n. 318, p.1-11, 2019. Disponível em: https://doi.org/10.1186/s12879-019-3940-4. Acesso em: 05 de maio de 2021. 
7- TEMPONI, Andrea Oliveira Dias et al. Ocorrência de casos de leishmaniose tegumentar americana: uma análise multivariada dos circuitos espaciais de produção, Minas Gerais, Brasil, 2007 a 2011. Cadernos de Saúde Pública, v. 34, n.2: e00165716, 2018. Disponível em: https://doi.org/10.1590/0102-311X00165716. Acesso em: 05 de maio de 2021.

8- PINTO, Mariana Olímpia Köhler Marra et al. Profile of American tegumentary leishmaniasis in transmission areas in the state of Minas Gerais, Brazil, from 2007 to 2017. BMC Infectious Diseases, London, v.20, n.163, 2020. Disponível em: https://doi.org/10.1186/s12879-020-4860-z. Acesso em: 05 de maio de 2021.

9- INSTITUTO BRASILEIRO DE GEOGRAFIA E ESTATÍSTICA (IBGE). Portal cidades. [Internet]. Disponível em: <https://cidades.ibge.gov.br/brasil/mg/montesclaros/panorama> Acesso em: 02 de setembro de 2020.

10- INSTITUTO BRASILEIRO DE GEOGRAFIA E ESTATÍSTICA (IBGE). Censo Demográfico 2010.

11- BRASIL. Ministério da Saúde. Conselho Nacional de Saúde. Resolução n o 466, de 12 de dezembro de 2012. Aprova diretrizes e normas regulamentadoras de pesquisas envolvendo seres humanos. Brasília, 2012.

12-SILVA, Patrick Leonardo Nogueira et. al. Características Epidemiológicas da Leishmaniose Tegumentar Americana no Norte de Minas Gerais. Revista Norte Mineira de Enfermagem, Montes Claros, v.2, n.1, p.43-50, 2014.

13- URSINE, Renata Luiz et al. American Tegumentary Leishmaniasis in an endemic municipality in the North of Minas Gerais State: spatial analysis and socioenvironmental factors. Revista do Instituto de Medicina Tropical de São Paulo, São Paulo, v.63, e2, 2021. Disponível em: https://doi.org/10.1590/S1678-9946202163002. Acesso em: 16 de fevereiro de 2021.

14- MARCHI, Melca Niceia Altoé de et al. Spatial analysis of leishmaniasis in Brazil: a systematized review. Revista do Instituto de Medicina Tropical de São Paulo, São Paulo, v. 61, e68, 2019. Disponível em: https://doi.org/10.1590/S16789946201961068. Acesso em: Acesso em: 10 de janeiro de 2021.

15-BRILHANTE, Andreia Fernandes et al. Epidemiological aspects of American cutaneous leishmaniasis (ACL) in an endemic area of forest extractivist culture in western Brazilian Amazonia. Revista do Instituto de Medicina Tropical de São Paulo, São Paulo, v.59, e12, 2017. Disponível em: https://doi.org/10.1590/S16789946201759012. Acesso em:

16-DETONI, Mariana Barbosa et al. Temporal and spatial distribution of American tegumentary leishmaniasis in north Paraná: 2010-2015. Revista da Sociedade Brasileira de Medicina Tropical, Uberaba, v. 52, e20180119, 2019. Disponível em: https://doi.org/10.1590/0037-8682-0119-2018. Acesso em: 12 de janeiro de 2021.

17-LOCKARD, Ryan D.; WILSON, Mary E.; RODRÍGUEZ, Nilda E. Sex-Related Differences in Immune Response and Symptomatic Manifestations to Infection with Leishmania Species. Journal of Immunology Research, v. 2019, n.4103819, 14 p., 2019. Disponível em: https://doi.org/10.1155/2019/4103819. Acesso em: 17 de maio de 2021.

18-GANGNEUX, Jean-Pierre et al. Recurrent American cutaneous leishmaniasis. Emerging infectious diseases, v.13, n.9, p.1436-8., 2007. Disponível em: https://doi.org/10.3201/eid1309.061446. Acesso em: 09 de junho de 2021. 
19- MENDONÇA, Mitzi G. et al. Persistence of Leishmania Parasites in Scars after Clinical Cure of American Cutaneous Leishmaniasis: Is There a Sterile Cure? The Journal of Infectious Diseases, v.189, n.6, p.1018-1023, 2004. Disponível em: https://doi.org/10.1086/382135. Acesso em: 18 de maio de 2021.

20-GRANGEIRO JUNIOR, Cícero Roberto Pinheiro et al. American cutaneous leishmaniasis in a northeast Brazilian city: clinical and epidemiological features. Revista da Sociedade Brasileira de Medicina Tropical, Uberaba, v.51, n.6, p.837-842, 2018. Disponível em: https://doi.org/10.1590/00378682-0504-2017. Acesso em: 17 de maio de 2021.

21-SALDANHA, Maíra G. et. al. Characterization of the Histopathologic Features in Patients in the Early and Late Phases of Cutaneous Leishmaniasis. The American Society of Tropical Medicine and Hygiene, v.96, n.3, p.645-652, 2021. Disponível em: https://doi.org/10.4269/ajtmh.16-0539. Acesso em: 24 de abril de 2021.

22-RODRIGUES, Alex Miranda et. al. Factors associated with treatmet failure of cutaneous leishmaniasis with meglumine antimoniate. Revista da Sociedade Brasileira de Medicina Tropical, Rio de Janeiro, v.39, n.2, p.139-145, 2006. Disponível em: http://dx.doi.org/10.1590/S0037-86822006000200001. Acesso em: 09 de março de 2021.

23- BRASIL. Ministério da Saúde. Secretaria de Vigilância em Saúde. Departamento de Vigilância Epidemiológica. Manual de recomendações para diagnóstico, tratamento e acompanhamento de pacientes com a coinfecção Leishmania-HIV- Brasília, Distrito Federal: Ministério da Saúde, 2011. 106 p.

24- OLIVEIRA, Romário de Sousa et. al. Ocorrência da coinfecção leishmaniose tegumentar americana/HIV no Estado do Maranhão. Revista Eletrônica Acervo Saúde, v.11, n.11: e487, 2019. Disponível em: https://doi.org/10.25248/reas.e487.2019. Acesso em: 19 de março de 2021. 